\title{
Fitness costs of adaptive chlorantraniliprole resistance in the Spodoptera exigua (Lepidoptera: Noctuidae)
}

\author{
Changwei Gong ${ }^{1}$, Xinge Yao ${ }^{1}$, Xuegui Wang ${ }^{1}$, Qunfang Yang ${ }^{1}$, Yuming Zhang ${ }^{1}$, and Litao \\ Shen ${ }^{1}$ \\ ${ }^{1}$ Sichuan Agricultural University - Chengdu Campus
}

August 19, 2020

\begin{abstract}
The beet armyworm, Spodoptera exigua, is a multifeeding insect pest, which has developed high resistance to chlorantraniliprole, a benzoylurea insecticide that targets on the ryanodine receptors (RyRs). However, few studies have been conducted on the highly resistant strain. Here, the resistant strain (SE-Sel) and sensitive strain (SE-Sus) were obtained by bidirectional screening for 6 generations. The potential oviposition and oviposition rate of the SE-Sel strain were dramatically lower than those of the SE-Sus strain, on the contrary the weights of prepupae and preadult were significantly increased. And the expression levels of vitellogenin $(\mathrm{SeVg})$ and its receptor $(\mathrm{SeVgR})$ in the SE-Sel strain were consistently lower than those in the SE-Sus strain. The RyRI4765M mutation and the upregulation of detoxification genes, such as SeABCOK, SeGST15, SeGSTZ2, SeCarEs1, CYP6AEW and SeCYP6AB10, contributed to the evolution of resistance to chlorantraniliprole. The RyRI4765M mutation could affect neuropeptide activation, and it conduced to the upregulated expression of the neuropeptide SeNPF and its receptor, SeNPFR, which could inhibit courtship behavior and reduce oviposition. And the neuropeptide SeNPF could influence the expression of juvenile hormone-binding protein and juvenile hormone diol kinase, and it led to the downregulated expression of $\mathrm{SeVg}$. Therefore, these results indicate that the fitness cost accompanied by chlorantraniliprole resistance in $\mathrm{S}$. exigua is related to the wicked evolution of RyR.
\end{abstract}

\section{Hosted file}

manuscript.pdf available at https://authorea.com/users/352117/articles/476488-fitnesscosts-of-adaptive-chlorantraniliprole-resistance-in-the-spodoptera-exigua-lepidopteranoctuidae 